Research Paper

\title{
Clinicopathological Implications of Human Papilloma Virus (HPV) LI Capsid Protein Immunoreactivity in HPVI6-Positive Cervical Cytology
}

\author{
Sung-Jong Lee ${ }^{1}$, Ah-Won Lee ${ }^{2}$, Chang-Suk Kang2 ${ }^{2}$ Jong-Sup Park ${ }^{3}$, Dong-Choon Park ${ }^{1}$, Eun-Young Ki³, \\ Keun-Ho Lee ${ }^{3}$, Joo-Hee Yoon ${ }^{1}$, Soo-Young Hur ${ }^{3}$, Tae-Jung Kim² ${ }^{\circledR}$ \\ 1. Department of Obstetrics and Gynecology, St. Vincent's Hospital, College of Medicine, The Catholic University of Korea, Suwon, Re- \\ public of Korea \\ 2. Department of Hospital Pathology, College of Medicine, The Catholic University of Korea, Seoul, Republic of Korea \\ 3. Department of Obstetrics and Gynecology, Seoul St. Mary's Hospital, College of Medicine, The Catholic University of Korea, Seoul, \\ Republic of Korea
}

\begin{abstract}
$\triangle$ Corresponding author: Tae-Jung Kim, Department of Hospital Pathology, Yeouido St. Mary's Hospital, 62 Yeouido-dong Yongdeungpo-gu, Seoul, Republic of Korea (Postal code: 150-010). Phone: +82-2-3779-2157; Fax: +82-2-783-6648; Email: kimecho@catholic.ac.kr

(c) Ivyspring International Publisher. This is an open-access article distributed under the terms of the Creative Commons License (http://creativecommons.org/ licenses/by-nc-nd/3.0/). Reproduction is permitted for personal, noncommercial use, provided that the article is in whole, unmodified, and properly cited.
\end{abstract}

Received: 2012.11.22; Accepted: 2013.12.11; Published: 2013.12.20

\begin{abstract}
Background: The objective of this study was to investigate the expression of human papilloma virus (HPV) LI capsid protein in abnormal cervical cytology with HPVI6 infection and analyze its association with cervical histopathology in Korean women.

Material and Methods: We performed immunocytochemistry for HPV LI in 475 abnormal cervical cytology samples from patients with HPVI6 infections using the Cytoactiv ${ }^{\circledR}$ HPV LI screening set. We investigated the expression of HPV LI in cervical cytology samples and compared it with the results of histopathological examination of surgical specimens.

Results: Of a total of 475 cases, 188 (39.6\%) were immunocytochemically positive and 287 (60.4\%) negative for HPV LI. The immunocytochemical expression rates of HPV LI in atypical squamous cells of unknown significance (ASCUS), low-grade squamous intraepithelial lesions (LSIL), high-grade squamous intraepithelial lesions (HSIL), and cancer were $21.8 \%, 59.7 \%, 19.1 \%$, and $0.0 \%$, respectively. LSIL exhibited the highest rate of HPV LI positivity. Of a total of 475 cases, the multiple-type HPV infection rate, including HPVI6, in HPV LI-negative cytology samples was $27.5 \%$, which was significantly higher than that in HPV LI-positive cytology samples $(p=0.037)$. The absence of HPV LI expression in ASCUS and LSIL was significantly associated with high-grade ( $\geq$ cervical intraepithelial neoplasia [CIN] 2) than low-grade ( $\leq \mathrm{CINI})$ histopathology diagnoses $(p<$ 0.05 ), but was not significantly different between HPVI 6 single and multiple-type HPV infections ( $p$ $>0.05$ ). On the other hand, among $188 \mathrm{HPV}$ LI-positive cases, $30.6 \%$ of multiple-type HPV infections showed high-grade histopathology diagnoses $(\geq \mathrm{CIN} 3)$, significantly higher than the percentage of HPVI6 single infections $(8.6 \%)(p=0.0004)$

Conclusions: Our study demonstrates that the expression of HPV LI is low in advanced dysplasia. Furthermore, the absence of HPV LI in HPVI6-positive low-grade cytology (i.e., ASCUS and LSIL) is strongly associated with high-grade histopathology diagnoses. The multiplicity of HPV infections may have an important role in high-grade histopathology diagnoses ( $\geq$ CIN3) in HPV LI-positive cases.
\end{abstract}

Key words: cervical cancer, cervical intraepithelial neoplasia, human papillomavirus, immunocytochemistry, cervical cytology 


\section{Introduction}

Cervical cancer is the second most common type of cancer in women worldwide [1] and the fourth most common female malignancy in Korea [2, 3]. Human papillomavirus (HPV) infection is a well-known prerequisite for the development of cervical cancer. Moreover, the integration of high-risk $\mathrm{HPV}$ is a key step in cervical neoplastic progression [4]. HPV type 16 (HPV16) is regarded as the most oncogenic in cervical carcinogenesis and is detectable in more than $50 \%$ of cervical cancers $[5,6]$. HPV testing in addition to a Papanicolaou smear has helped reduce the development of grade 2 and 3 cervical intraepithelial neoplasia (CIN) and cancer [7]. Furthermore, the epidemiological and biological effects of multiple-type HPV infections in the presence of high-risk HPV infections have been documented with the increasing administration of HPV vaccines [8].

HPV L1 capsid protein comprises $90 \%$ of HPV viral surface proteins and is typically expressed during the late phase of viral replication [9]. Thus far, most immunocytochemical studies on the HPV L1 capsid protein have investigated the predictive role of the protein in low-grade squamous intraepithelial lesions (LSIL) and atypical squamous cells of unknown significance (ASCUS) [10-12]. Unfortunately, few comprehensive studies have investigated HPV L1 protein immunoreactivity in abnormal cytology, including high-grade squamous intraepithelial lesions (HSIL) and cancer according to the multiplicity of HPV infections in the presence of HPV16.

This study investigated the expression of HPV L1 in abnormal cervical cytology with HPV16 infection and analyzed its association with HPV multiplicity and cervical histopathology diagnoses in Korean women.

\section{Materials and methods}

\section{Patient selection}

We analyzed 475 abnormal cervical cytology samples from patients with HPV16 infection confirmed by HPV DNA genotyping at Seoul St. Mary's Hospital, The Catholic University of Korea, Seoul, Korea, from January 2007 to December 2012. The cervical cytology samples were gently scraped from the exocervix and endocervix with a cervical brush. Cytological diagnosis was carried out according to the 2001 Bethesda reporting system [13], and HPV type was determined by a DNA genotyping polymerase chain reaction-based DNA microarray system (Mygene, Seoul, Korea) that detected 16 types of high-risk HPV $(16,18,31,33,35,39,45,51,52,53,54$, $56,58,59,66$, and 68$)$ and 8 types of low-risk HPV (6,
11, 34, 40, 42, 43, 44, and 70) [14]. The results of tissue diagnosis were obtained from records of patients who underwent colposcopic biopsy, loop electrosurgical excision procedures, or total hysterectomy. The following parameters were collected from medical records: age, cervical cytology, HPV infection type, and results of cervical histopathology of surgical specimens.

The study design, data collection, and analysis followed the principles of the Declaration of Helsinki. This study was approved by the Institutional Review Board of The Catholic University of Korea.

\section{Immunocytochemistry}

The immunocytochemical reaction for HPV L1 capsid protein in abnormal cervical cytology was performed using the Cytoactiv ${ }^{\circledR}$ HPV L1 screening set (Cytoimmun Diagnostics GmbH, Pirmasens, Germany) according to the manufacturer's protocols. In brief, the coverslip and mounting medium of Papanicolaou-stained cervical smear slides in xylene were separated, and the slides were washed using absolute alcohol to eliminate the remaining xylene. To retrieve the antigen, $0.01 \mathrm{~mol} / \mathrm{L}$ citrate buffer $(\mathrm{pH}$ 6.0) was used, and the sample was heated in a microwave vacuum histoprocessor (RHS-1; Milestone, Bergamo, Italy) at a controlled final temperature of $121^{\circ} \mathrm{C}$ for 15 minutes. The slides were kept at room temperature for 10 minutes to cool down. Afterwards, one droplet of HPV L1 capsid antibody was applied to the cervical smear slides, and the slides were incubated for 1 hour at room temperature. A drop of detection reagent (ready to use) was applied and incubated for 10 minutes. Next, a drop of chromogen solution (3-amino-9-ethylcarbazole, ready to use) was applied to the slide for 5 minutes. Finally, Mayer's hematoxylin counterstaining was applied to the samples. We included at least 1 positive control slide supplied by the manufacturer in each batch. The slides were examined by 2 pathologists (TJ Kim and AW Lee) and scored using a 3-step algorithm [15]: (1) the adequacy of the specimen was determined according to the cervical cytology criteria of the 2001 Bethesda System; (2) whether there was red nuclear staining in the squamous cells was determined; and (3) whether the stained cells showed abnormalities that conformed to the diagnostic criteria was determined.

\section{Statistical analysis}

We investigated the expression of HPV L1 in cervical cytology and compared it with that of cervical histopathology. Analysis of variance, Pearson's $X^{2}$, and Fisher's exact tests were used to determine the associations between the immunocytochemical expression of HPV L1 capsid and other variables. All 
calculations were performed using SAS version 8.0 (SAS institute Inc., Cary, NC, USA). The level of significance was set at $p<0.05$.

\section{Results}

The immunocytochemical expression of the HPV L1 capsid protein was observed mainly in the nuclei of cervical cells (Fig.1). Of a total of 475 cases, 188 (39.6\%) were immunocytochemically positive and 287 $(60.4 \%)$ negative for HPV L1. Three hundred sixty (75.8\%) patients were infected with only HPV16, whereas $115(24.2 \%)$ patients exhibited multiple-type HPV infections, including HPV16. Further, the patients comprising the cancer group were older (mean age: 56.6 years) than those of any other groups, with a statistically significant difference $(p<0.0001)$ (Table $1)$.

The immunocytochemical expression rates of HPV L1 in ASCUS, LSIL, HSIL, and cancer were $21.8 \%, 59.7 \%, 19.1 \%$, and $0.0 \%$, respectively. LSIL exhibited the highest rate of HPV L1 positivity among the 4 groups $(p<0.0001)$. Although the expression of L1 tended to be negatively associated with the cytology grades, a high percentage $(78.2 \%)$ of samples without L1 were observed in cases of ASCUS (Table 1). HSIL showed the highest rate $(38.3 \%)$ of HPV16 multiple infections, and cancer cytology expressed the lowest rate $(7.3 \%)$ of multiple-type HPV infections $(p$ $<0.0001$ ) (Table 1).



(a) ASCUS

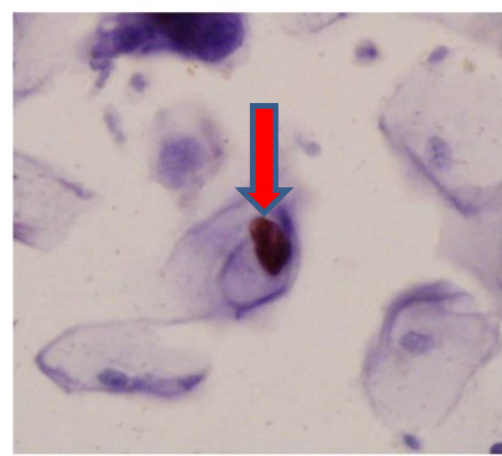

(b) LSIL

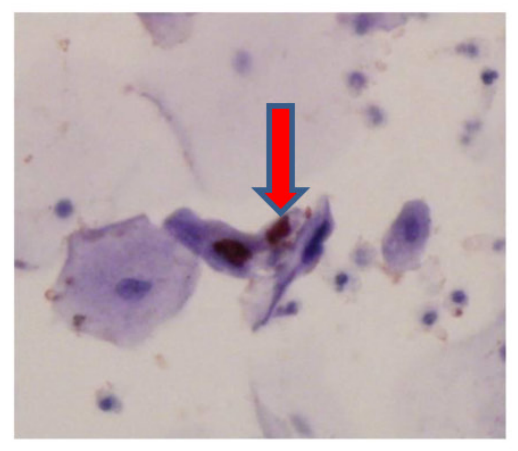

(c) HSIL

Fig. I. The immunocytochemical expression of HPV LI capsid protein in the nuclei of cervical cells.

Table I. Clinicopathological parameters and HPV LI protein expression of HPV I6-positive abnormal cervical cytology (N =475).

\begin{tabular}{|c|c|c|c|c|c|c|c|c|c|c|c|}
\hline & $\begin{array}{l}\text { Total } \\
(\mathrm{N}=475)\end{array}$ & & $\begin{array}{l}\text { ASCUS }{ }^{\dagger} \\
(\mathrm{n}=87)\end{array}$ & & $\begin{array}{l}\text { LSIL } \ddagger \\
(\mathrm{n}=253)\end{array}$ & & $\begin{array}{l}\text { HSIL } \\
(\mathrm{n}=94)\end{array}$ & & $\begin{array}{l}\text { Cancer } \\
(\mathrm{n}=41)\end{array}$ & & \\
\hline & number & $(\%)$ & number & $(\%)$ & number & $(\%)$ & number & $(\%)$ & number & $(\%)$ & $p \pi$ \\
\hline \multicolumn{12}{|c|}{$\mathrm{HPV}^{*}$ L1 capsid immunocytochemistry } \\
\hline Negative & 287 & $(60.4)$ & 68 & $(78.2)$ & 102 & $(40.3)$ & 76 & $(80.9)$ & 41 & $(100.0)$ & $<0.0001$ \\
\hline Positive & 188 & $(39.6)$ & 19 & $(21.8)$ & 151 & $(59.7)$ & 18 & $(19.1)$ & 0 & $(0)$ & \\
\hline \multicolumn{12}{|c|}{ HPV 16 infection } \\
\hline Single & 360 & $(75.8)$ & 60 & $(69.0 \%)$ & 204 & $(80.6 \%)$ & 58 & $(61.7 \%)$ & 38 & $(92.7 \%)$ & $<0.0001$ \\
\hline Multiple & 115 & $(24.2)$ & 27 & $(31.0 \%)$ & 49 & $(19.4 \%)$ & 36 & $(38.3 \%)$ & 3 & $(7.3 \%)$ & \\
\hline \multicolumn{12}{|l|}{ Age (years) } \\
\hline Mean age & 38.7 & & 35.6 & & 36.6 & & 39.5 & & 56.6 & & $<0.0001$ \\
\hline $20-29$ & 129 & $(26.6)$ & 23 & (26.4) & 83 & $(32.8)$ & 23 & $(24.5)$ & 0 & $(0)$ & $<0.0001$ \\
\hline $30-39$ & 163 & $(33.6)$ & 40 & $(46.0)$ & 83 & $(32.8)$ & 34 & $(36.2)$ & 6 & $(14.6)$ & \\
\hline $40-49$ & 123 & $(25.4)$ & 23 & $(26.4)$ & 68 & $(26.9)$ & 20 & (21.3) & 12 & (29.3) & \\
\hline$\geq 50$ & 60 & (12.4) & 1 & $(1.2)$ & 19 & (7.5) & 17 & $(18.0)$ & 23 & $(56.1)$ & \\
\hline
\end{tabular}

*HPV: human papillomavirus

†ASCUS: atypical squamous cells of undetermined significance

‡LSIL: low-grade squamous intraepithelial lesion

§HSIL: high-grade squamous intraepithelial lesion

๑ Evaluated by Fisher's Exact Test and $t$-test 
Among 87 cases of ASCUS, 23 (26.4\%) cases were $\geq \mathrm{CIN} 2$ and $10(11.5 \%)$ cases were $\geq \mathrm{CIN} 3$. Further, 64 cases $(73.6 \%)$ of ASCUS showed low-grade histopathology results. Cervical cancer was absent in the ASCUS cases. In 87 cases of ASCUS, HPV L1-negative cases showed a significantly higher trend towards $\geq$ CIN2 progression than did HPV L1-positive cases ( $p$ = 0.002; OR: 20.1; 95\% confidence interval (CI): 1.1 to $348.8)$. Among 253 cases of LSIL, $70(27.7 \%)$ cases were $\geq \mathrm{CIN} 2$ and $32(12.6 \%)$ were $\geq \mathrm{CIN} 3$. In 253 cases of LSIL, HPV L1-negative cases showed a significant association with $\geq \mathrm{CIN} 2$ than did HPV L1-positive cases ( $p=0.001$; OR: 4.8 ; 95\% CI: 2.6 to 8.6). Moreover, 25 HPV L1-negative cases $(56.9 \%)$ showed a significant relationship with $\geq \mathrm{CIN} 3$ compared to HPV L1-positive cases ( $p=0.001$; OR: 6.7; 95\% CI: 2.7 to 16.1). Among 94 cases of HSIL, 91 (96.8\%) cases were $\geq$ CIN2 and $85(90.4 \%)$ cases were $\geq$ CIN3. In HSIL, HPV L1 expression was not associated with a tendency of progression to high-grade pathology. All 41 cancer cases $(100 \%)$ showed HPV L1 negativity and $\geq$ CIN3 in cervical pathology (Table 2).

Of a total of 475 cases, the rate of multiple infections with HPV16 in HPV L1-negative cytology was $27.5 \%$, which was significantly higher than that in HPV L1-positive cytology ( $p=0.037$ ) (Fig. 2).
Table 3 illustrates the relationship between HPV L1 expression and histopathology according to the multiplicity of HPV16 infections. With regard to 287 HPV L1-negative cases, the multiplicity of HPV16 infections did not exhibit a significant role in discriminating between higher- and lower-grade histopathology results $(\geq \mathrm{CIN} 2$ versus $\leq \mathrm{CIN} 1, p=0.7663$; $\geq$ CIN3 versus $\leq$ CIN2, $p=0.0926$ ). However, among 188 HPV L1-positive cases, multiple-type HPV infections showed a significant association with high-grade histopathology results $(\geq \mathrm{CIN} 3)(p=0.0004$; OR: 4.7 ; $95 \%$ CI: 1.7 to 12.8 ) (Table 3).

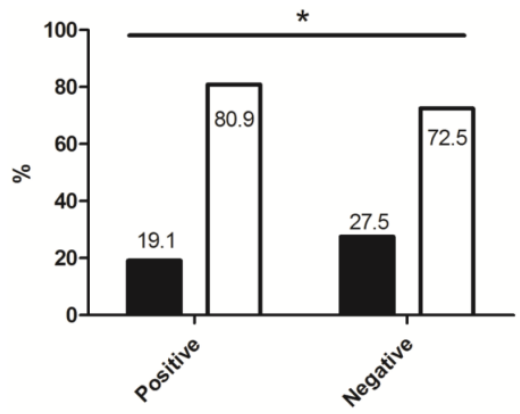

HPV L1 capsid immunochemistry

Fig. 2. The association between multiplicity of HPV 16 infection in abnormal cervical cytology and HPV LI capsid protein expression. *: HPV LI-negative cytology showed a significant relationship with multiple infections including HPV type $16(p=0.037)$.

Table 2. Association of HPV LI capsid protein expression in HPVI6-positive cytology and cervical histopathology $(\mathrm{N}=475)$.

\begin{tabular}{|c|c|c|c|c|c|c|c|c|c|c|c|c|c|}
\hline & & & & & \multicolumn{6}{|c|}{$\begin{array}{l}\mathrm{HPV}^{\star} \mathrm{L} 1 \text { capsid in cytology } \\
(\mathrm{N}=475)\end{array}$} & \multicolumn{3}{|c|}{$\begin{array}{l}\text { HPV L1 capsid in cytology } \\
(\mathrm{N}=475)\end{array}$} \\
\hline & & & & & $\begin{array}{l}\text { Positive } \\
(\mathrm{n}=188)\end{array}$ & $\begin{array}{l}\text { Negative } \\
(\mathrm{n}=287)\end{array}$ & & & & & $\begin{array}{l}\text { Positive } \\
(\mathrm{n}=188)\end{array}$ & $\begin{array}{l}\text { Negative } \\
(\mathrm{n}=287)\end{array}$ & \\
\hline Cytology & Number & $\begin{array}{l}\text { Histo- } \\
\text { pathology }\end{array}$ & Number & $(\%)$ & Number & Number & $p \pi$ & $\begin{array}{l}\text { Histo- } \\
\text { pathology }\end{array}$ & Number & $(\%)$ & Number & Number & $p \pi$ \\
\hline \multirow[t]{2}{*}{ ASCUSt } & 87 & $\leq \mathrm{CIN} 1$ & 64 & (73.6) & 19 & 45 & 0.002 & $\leq \mathrm{CIN} 2$ & 77 & $(88.5)$ & 19 & 58 & 0.109 \\
\hline & & $\geq \mathrm{CIN} 2$ & 23 & (26.4) & 0 & 23 & & $\geq \mathrm{CIN} 3$ & 10 & (11.5) & 0 & 10 & \\
\hline \multirow[t]{2}{*}{ LSIL $\ddagger$} & 253 & $\leq \mathrm{CIN} 1$ & 183 & (72.3) & 128 & 55 & $<0.001$ & $\leq \mathrm{CIN} 2$ & 221 & $(87.4)$ & 144 & 77 & $<0.001$ \\
\hline & & $\geq \mathrm{CIN} 2$ & 70 & $(27.7)$ & 23 & 47 & & $\geq \mathrm{CIN} 3$ & 32 & (12.6) & 7 & 25 & \\
\hline \multirow[t]{2}{*}{ HSIL $\$$} & 94 & $\leq \mathrm{CIN} 1$ & 3 & (3.2) & 1 & 2 & 0.475 & $\leq \mathrm{CIN} 2$ & 9 & $(9.6)$ & 1 & 8 & 1.000 \\
\hline & & $\geq \mathrm{CIN} 2$ & 91 & (96.8) & 17 & 74 & & $\geq \mathrm{CIN} 3$ & 85 & (90.4) & 17 & 68 & \\
\hline \multirow[t]{2}{*}{ Cancer } & 41 & $\leq \mathrm{CIN} 1$ & 0 & $(0)$ & 0 & 0 & NA।I & $\leq \mathrm{CIN} 2$ & 0 & (0) & 0 & 0 & NA \\
\hline & & $\geq \mathrm{CIN} 2$ & 41 & (100) & 0 & 41 & & $\geq \mathrm{CIN} 3$ & 0 & $(100)$ & 0 & 41 & \\
\hline
\end{tabular}

"HPV: human papillomavirus

tASCUS: atypical squamous cells of undetermined significance

‡LSIL: low-grade squamous intraepithelial lesions

sHSIL: high-grade squamous intraepithelial lesions

IINA: not available, ^ Evaluated by Fisher's Exact Test

Table 3. Association of HPV LI capsid protein expression with underlying histopathology according to multiplicity of HPV infection in HPV 16-positive abnormal cervical cytology $(\mathrm{N}=475)$.

\begin{tabular}{|c|c|c|c|c|c|c|c|c|c|c|c|c|c|c|c|}
\hline & & & & & & \multicolumn{5}{|c|}{ Histopathology } & \multicolumn{5}{|c|}{ Histopathology } \\
\hline & & & & & & $\leq \mathrm{CIN} 1$ & & $\geq$ CIN2 & & & $\leq \mathrm{CIN} 2$ & & $\geq \mathrm{CIN} 3$ & & \\
\hline $\mathrm{HPV}^{*} \mathrm{L1}$ & Number & $(\%)$ & HPV type & Number & $(\%)$ & Number & $(\%)$ & Number & $(\%)$ & $p^{\dagger}$ & Number & $(\%)$ & Number & $(\%)$ & $p^{\dagger}$ \\
\hline \multirow[t]{2}{*}{ Negative } & 287 & $(60.4)$ & Single & 208 & $(72.5)$ & 75 & $(36.1)$ & 133 & $(63.9)$ & 0.7663 & 98 & $(47.1)$ & 110 & $(52.9)$ & 0.0926 \\
\hline & & & Multiple & 79 & $(27.5)$ & 27 & $(34.2)$ & 52 & $(65.8)$ & & 46 & $(58.2)$ & 33 & $(41.8)$ & \\
\hline \multirow[t]{2}{*}{ Positive } & 188 & $(39.6)$ & Single & 152 & $(80.9)$ & 123 & $(80.9)$ & 29 & $(19.1)$ & 0.1303 & 139 & $(91.4)$ & 13 & (8.6) & 0.0004 \\
\hline & & & Multiple & 36 & $(19.1)$ & 25 & $(69.4)$ & 11 & $(30.6)$ & & 25 & $(69.4)$ & 11 & $(30.6)$ & \\
\hline
\end{tabular}

*HPV: human papillomavirus

† Evaluated by $\chi^{2}$ test 


\section{Discussion}

Our immunocytochemistry study showed that the expression of the HPV L1 capsid protein was higher in LSIL than ASCUS, HSIL, and cancer. Interestingly, all cancer cases tested negative for HPV L1. In addition, the absence of HPV L1 in low-grade cytology such as ASCUS and LSIL was significantly related to high-grade cervical pathology ( $\geq \mathrm{CIN} 2)$ compared to cases of HPV L1 positivity.

In low-grade (CIN1) cervical tissue, HPV usually exists in an episomal state in the host nucleus and proceeds into the productive cycle. The productive cycle is composed of HPV infection of the basement membrane and transcription, translation, and assembly of the L1 capsomere in the epithelium [16]. Finally, it produces L1 in the superficial layers of the cervical epithelium $[17,18]$. On the other hand, in cases with high-grade pathology such as CIN2 and CIN3, the E2 open reading frame of HPV DNA is frequently disrupted and integrated into the human genome [19, 20]. The loss of E2 inhibitory function on the E6 and E7 open reading frames selectively produces E6 and E7 oncoproteins and interrupts normal cell cycles by suppression of the tumor suppressor proteins p53 and $\mathrm{Rb}$. In abortive HPV cycles of high-grade lesions, HPV
L1 cannot be immunocytochemically detected at the superficial epithelium [21] (Fig. 3).

Reports on the correlation between cytology and cervical histopathology results have indicated that $90 \%$ of LSIL cases exhibited CIN1 and cervicitis, and $10 \%$ of LSIL cases exhibited $\geq$ CIN2 histopathology [22]. Regarding the patterns of HPV L1 capsid immunocytochemistry, HPV L1 expression tended to decrease with higher cytological grade even though the positive rates were variable [19, 22-24]. For HPV L1 immunocytochemistry, we showed that HPV L1-negative LSILs exhibited a higher prevalence of $\geq$ CIN2 pathology than did HPV L1-positive LSIL cases. Among the 151 HPV L1-positive LSIL cases, 128 $(84.7 \%)$ had CIN1 and cervicitis and 23 cases had $(15.3 \%) \geq$ CIN2 histopathology. Meanwhile, among 102 HPV L1-negative LSIL cases, 55 (53.9\%) had CIN1 and cervicitis and 47 (46.1\%) had $\geq$ CIN2 pathology. These patterns were similar to those observed in previous studies [22]. On the other hand, regarding HSILs and cancer cytology, the correlation between cytology and histopathology results were not significantly different with respect to HPV L1 immunocytochemical reactivity.

Cervical epithelium

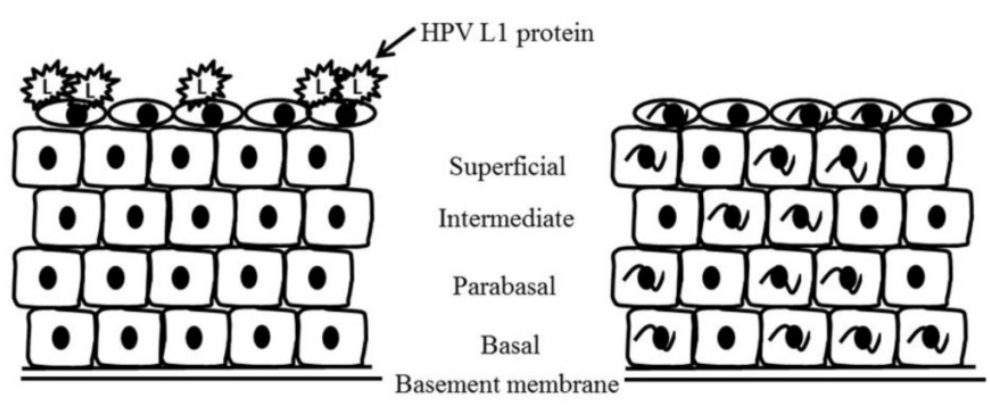

Human nucleus

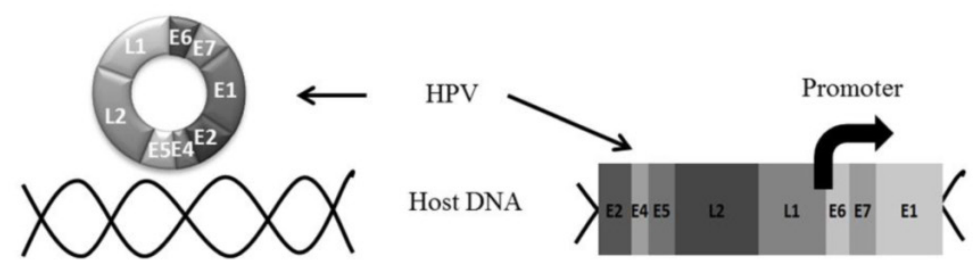

Cytology
Pathology
HPV DNA status
HPV L1 protein production at
superficial layer

LSIL

HSIL, Cancer

CIN 1

CIN2, CIN 3, Cancer

Episome

Integration

Much

Less

Fig. 3. The comparison of HPV LI capsid protein expression between low-grade and high-grade severity of cervical epithelium. The productive cycle is observed in low-grade lesion and abortive cycle of HPV can be seen in high-grade pathology. HPV LI capsid protein is more frequently produced in the superficial epithelium of LSIL than HSIL. HPV integration into host genome can be identified in high-grade lesion of cervix. 
In this study, we wanted to explore the rate of HPV L1 expression in ASCUS cytology. Because ASCUS has shown a wide spectrum of histopathology from reactive change to carcinoma, it was also very difficult to understand the characteristics of ASCUS. Usually $70 \%$ of ASCUS cases show low-grade pathology such as CIN1 after HPV infection. Although HPV L1-negative cases tend to correlate with the cytology grade, a relatively low HPV L1 expression rate was observed in cases of ASCUS. Interestingly, all 23 cases with $\geq \mathrm{CIN} 2$ histopathology were HPV L1-negative on immunocytochemical analysis. Ungureanu et al. reported that the HPV L1 positivity rates in ASCUS, LSIL, HSIL, and cancer cytology were $33.3 \%, 50.0 \%, 18.5 \%$, and $0 \%$, respectively [23]. Those results were very similar to ours. HPV L1 negativity is associated with low amounts of HPV L1 production, which could imply 2 different conditions of HPV infection in ASCUS. We postulated that these conditions could be observed during (1) viral entry or persistence before viral synthesis in the HPV life cycle and (2) HPV DNA integration in the human genome [24]. The characteristics of HPV L1 expression in ASCUS may be slightly different from that of LSIL, although ASCUS and LSIL have been generally regarded as minor abnormalities in the Bethesda cytology system. Further translational research will be necessary to identify the nature of HPV L1 negativity more clearly in ASCUS.

In the natural history of cervical cytology, $80 \%$ of LSIL cases spontaneously regress within 24 months [25]. However, $40 \%$ of LSILs infected with HPV16 progress to CIN3 or cancer [26]. In our study, among 253 patients with HPV16-positive LSILs, 32 cases $(12.6 \%)$ were $\geq$ CIN3. Similarly, Matsumoto et al. [27] reported that infection with high-risk HPV types, including $16,18,31,33,35,52$, and 58, confers a higher risk for $\geq \mathrm{CIN} 3$ pathology in LSIL than infection with the other high-risk HPV types. Hosaka et al. [28] reported that the incidence of CIN3 according to HPV status is $19.1 \%$ in cases of infection with high-risk HPV including HPV 16, 18, and 33, but just $4.2 \%$ in cases of infection with other high-risk HPV types, including 31, 35, 52, and 58. Kahn et al. [29] reported that HPV16 and HPV18 infection observed in negative, equivocal, and mildly abnormal cytology is associated with a higher risk for incidence of $\geq \mathrm{CIN} 3$ pathology. Accordingly, we collected samples with abnormal cervical cytology with HPV16 infection and investigated their histopathology results according to HPV L1 immunocytochemistry reactivity. Yoshida et al. [21] reported HPV L1 positivity in 30\% of LSIL and $12 \%$ of HSIL cases, a trend similar to that observed in the present study. These results suggested that HPV L1 positivity in LSILs with HPV16 infection might be attributable to the effect of the L1 protein on keratinocytes infected with other high-risk HPV types $[23,29]$.

HPV16 is a member of the human alphapapillomavirus-9 group [30] and has shown to confer a significantly higher risk of developing high-grade CIN lesions or invasive cancers in combination with other HPV types [31]. Interestingly, the rate of HPV L1 expression in higher-grade histopathology $(\geq \mathrm{CIN} 2$ versus $\leq \mathrm{CIN} 1$ and $\geq \mathrm{CIN} 3$ versus $\leq \mathrm{CIN} 2$ ) was not significantly different between HPV16 single infections and multiple-type HPV infections in the presence of HPV16 infection. However, cases that were HPV L1-positive with multiple-type HPV infections were significantly associated with high-grade histopathology ( $\geq$ CIN3) results. HPV L1-positive cases with multiple-type HPV infections comprised $30.6 \%$ of $\geq \mathrm{CIN} 3$ cases, while HPV L1-positive cases with HPV16 single infections accounted for only $8.6 \%$ of $\geq \mathrm{CIN} 3$ cases. Multiple-type HPV infections seemed to play an important role in progression to high-grade cervical lesions in HPV L1-positive cases.

From an immunological perspective, HPV L1 is immunogenic to $\mathrm{CD}^{+}$and $\mathrm{CD} 4^{+} \mathrm{T}$ cells, which play important roles in cellular immunity [32]. HPV L1-negative LSIL is reportedly more likely to demonstrate disease progression than L1-positive cases are; this suggests that HPV L1 has a beneficial immunological role in the prevention of disease progression [33]. Therefore, the absence of HPV L1 after HPV integration into the host genome could be associated with immunologic evasion in higher-grade lesions $[32,34]$. The presence of HPV L1 could be an indicator of a defense status in HPV infection [35]. Cervical cytology usually performed on the superficial layer of the cervix in a cancer screening test has shown high rates of false negativity and requires an adjuvant test for an accurate result. Therefore, HPV L1 tests that reflect the HPV productive cycle and the relationship between HPV integration and host genome in cervical epithelium will be more informative to understand cervical immunology.

The present study had some limitations. First, this was a retrospective cross-sectional study; therefore, the relationship between the HPV lifecycle and cervical neoplasia could not be fully understood solely on the basis of HPV L1 expression in the superficial layer. Second, this study did not include the effect of low-risk HPV on HPV L1 immunocytochemistry; future studies on low-risk HPV might be necessary. Third, in cases of low-grade cervical cytology with high-grade histopathology results, misclassification or sampling error of cytology specimens might be a cause for misinterpretation of immunocytochemistry results. Despite these limitations, to the best of our 
knowledge, this is the first study to examine the immunocytochemistry of HPV L1 according to HPV16-positive cytology, as well as the association between cervical cytology and histopathology according to the multiplicity of HPV16 infections.

In summary, our study demonstrated that the expression of HPV L1 was low in cases of advanced dysplasia. Furthermore, the absence of HPV L1 in HPV16-positive samples with low-grade cytology (i.e., ASCUS and LSIL) was strongly associated with high-grade histopathology results. This trend was not significantly different between the HPV16 single infection group and the multiple-type HPV infection group. On the other hand, multiple-type HPV infections were significantly associated with $\geq \mathrm{CIN} 3$ histopathology in HPV L1-positive patients. In future translational research, investigation of the association between HPV L1 and HPV integration according to the severity of cervical pathology and the multiplicity of HPV infection might be useful to understand clearly the mechanism of HPV productive and abortive cycles in cervical epithelium.

\section{Abbreviations}

ASCUS: atypical squamous cells of unknown significance; CIN: cervical intraepithelial neoplasia; HPV: human papillomavirus; HSIL: high-grade squamous intraepithelial lesion; LSIL: low-grade squamous intraepithelial lesion.

\section{Acknowledgements}

This work was supported by Department of Obstetrics and Gynecology, and Hospital Pathology, College of Medicine, The Catholic University of Korea. And it was also supported by the research grant from St. Vincent's Hospital.

\section{Competing Interests}

The authors have declared that no competing interest exists.

\section{References}

1. Karabulut A, Alan T, Ali Ekiz M, Iritas A, Kesen Z, Yahsi S. Evaluation of cervical screening results in a population at normal risk. Int J Gynaecol Obstet. 2010;110:40-2.

2. Shin HR, Jung KW, Won YJ, et al. National cancer incidence for the year 2002 in Korea. Cancer Res Treat. 2007;39:139-49.

3. Lim MC, Moon EK, Shin A, et al. Incidence of cervical, endometrial, and ovarian cancer in Korea, 1999-2010. J Gynecol Oncol. 2013;24:298-302.

4. Longworth MS, Laimins LA. Pathogenesis of human papillomaviruses in differentiating epithelia. Microbiol Mol Biol Rev. 2004;68:362-72.

5. Bosch FX, Manos MM, Munoz N, et al. Prevalence of human papillomavirus in cervical cancer: a worldwide perspective. International biological study on cervical cancer (IBSCC) Study Group. J Natl Cancer Inst. 1995;87:796-802.

6. Nishio S, Fujii T, Nishio H, et al. p16(INK4a) immunohistochemistry is a promising biomarker to predict the outcome of low grade cervical intraepithelial neoplasia: comparison study with HPV genotyping. J Gynecol Oncol. 2013;24:215-21.

7. Naucler P, Ryd W, Tornberg S, et al. Human papillomavirus and Papanicolaou tests to screen for cervical cancer. N Engl J Med. 2007;357:1589-97.

8. Dickson EL, Vogel RI, Bliss RL, Downs LS, Jr. Multiple-type human papillomavirus (HPV) infections: a cross-sectional analysis of the prevalence of specific types in 309,000 women referred for HPV testing at the time of cervical cytology. Int J Gynecol Cancer. 2013;23:1295-302.
9. Gu W, Ding J, Wang X, et al. Generalized substitution of isoencoding codons shortens the duration of papillomavirus L1 protein expression in transiently gene-transfected keratinocytes due to cell differentiation. Nucleic Acids Res. 2007;35:4820-32.

10. Lee SJ, Lee AW, Kim TJ, et al. Correlation between immunocytochemistry of human papilloma virus L1 capsid protein and behavior of low-grade cervical cytology in Korean women. J Obstet Gynaecol Res. 2011;37:1222-8.

11. Negri G, Bellisano G, Zannoni GF, et al. p16 ink4a and HPV L1 immunohistochemistry is helpful for estimating the behavior of low-grade dysplastic lesions of the cervix uteri. Am J Surg Pathol. 2008;32:1715-20.

12. Griesser $\mathrm{H}$, Sander $\mathrm{H}$, Walczak $\mathrm{C}$, Hilfrich RA. HPV vaccine protein $\mathrm{L} 1$ predicts disease outcome of high-risk HPV+ early squamous dysplastic lesions. Am J Clin Pathol. 2009;132:840-5.

13. Solomon D, Davey D, Kurman R, et al. The 2001 Bethesda System: terminology for reporting results of cervical cytology. JAMA. 2002;287:2114-9.

14. Bae JH, Kim CI, Park TC, Namkoong SE, Park JS. Persistence of human papillomavirus as a predictor for treatment failure after loop electrosurgical excision procedure. Int J Gynecol Cancer. 2007;17:1271-7.

15. Siddiqui MT, Hornaman K, Cohen C, Nassar A. ProEx C immunocytochemistry and high-risk human papillomavirus DNA testing in papanicolaou tests with atypical squamous cell (ASC-US) cytology: correlation study with histologic biopsy. Arch Pathol Lab Med. 2008;132:1648-52.

16. Resnick RM, Cornelissen MT, Wright DK, et al. Detection and typing of human papillomavirus in archival cervical cancer specimens by DNA amplification with consensus primers. J Natl Cancer Inst. 1990;82:1477-84.

17. Middleton $\mathrm{K}$, Peh $\mathrm{W}$, Southern $\mathrm{S}$, et al. Organization of human papillomavirus productive cycle during neoplastic progression provides a basis for selection of diagnostic markers. J Virol. 2003;77:10186-201.

18. Doorbar J. The papillomavirus life cycle. J Clin Virol. 2005;32 Suppl 1:S7-15.

19. Klaes R, Woerner SM, Ridder R, et al. Detection of high-risk cervical intraepithelial neoplasia and cervical cancer by amplification of transcripts derived from integrated papillomavirus oncogenes. Cancer Res. 1999;59:6132-6.

20. Vinther J, Rosenstierne MW, Kristiansen K, Norrild B. The $3^{\prime}$ region of human papillomavirus type 16 early mRNAs decrease expression. BMC Infect Dis. 2005;5:83.

21. Yoshida T, Sano T, Kanuma T, et al. Immunochemical analysis of HPV L1 capsid protein and p16 protein in liquid-based cytology samples from uterine cervical lesions. Cancer. 2008;114:83-8.

22. Shidham VB, Kumar N, Narayan R, Brotzman GL. Should LSIL with ASC-H (LSIL-H) in cervical smears be an independent category? A study on SurePath specimens with review of literature. Cytojournal. 2007;4:7.

23. Ungureanu C, Socolov D, Anton G, Mihailovici MS, Teleman S. Immunocytochemical expression of p16INK4a and HPV L1 capsid proteins as predictive markers of the cervical lesions progression risk. Rom J Morphol Embryol. 2010;51:497-503.

24. Doorbar J. Molecular biology of human papillomavirus infection and cervical cancer. Clin Sci (Lond). 2006;110:525-41.

25. Lee SS, Collins RJ, Pun TC, Cheng DK, Ngan HY. Conservative treatment of low grade squamous intraepithelial lesions (LSIL) of the cervix. Int J Gynaecol Obstet. 1998;60:35-40.

26. Castle PE, Solomon D, Schiffman M, Wheeler CM. Human papillomavirus type 16 infections and 2-year absolute risk of cervical precancer in women with equivocal or mild cytologic abnormalities. J Natl Cancer Inst. 2005;97:1066-71.

27. Matsumoto K, Oki A, Furuta R, et al. Predicting the progression of cervical precursor lesions by human papillomavirus genotyping: a prospective cohort study. Int J Cancer. 2011;128:2898-910.

28. Hosaka M, Fujita H, Hanley SJ, et al. Incidence risk of cervical intraepithelial neoplasia 3 or more severe lesions is a function of human papillomavirus genotypes and severity of cytological and histological abnormalities in adult Japanese women. Int J Cancer. 2013;132:327-34.

29. Khan MJ, Castle PE, Lorincz AT, et al. The elevated 10-year risk of cervical precancer and cancer in women with human papillomavirus (HPV) type 16 or 18 and the possible utility of type-specific HPV testing in clinical practice. J Natl Cancer Inst. 2005;97:1072-9.

30. Burk RD, Harari A, Chen Z. Human papillomavirus genome variants. Virology. 2013;445:232-43.

31. Trottier H, Mahmud S, Costa MC, et al. Human papillomavirus infections with multiple types and risk of cervical neoplasia. Cancer Epidemiol Biomarkers Prev. 2006;15:1274-80.

32. Steele JC, Roberts S, Rookes SM, Gallimore PH. Detection of CD4(+)- and $\mathrm{CD} 8(+)-\mathrm{T}$-cell responses to human papillomavirus type 1 antigens expressed at various stages of the virus life cycle by using an enzyme-linked immunospot assay of gamma interferon release. J Virol. 2002;76:6027-36.

33. Griesser H, Sander H, Hilfrich R, Moser B, Schenck U. Correlation of immunochemical detection of HPV L1 capsid protein in pap smears with regression of high-risk HPV positive mild/moderate dysplasia. Anal Quant Cytol Histol. 2004;26:241-5.

34. Melsheimer P, Kaul S, Dobeck S, Bastert G. Immunocytochemical detection of HPV high-risk type L1 capsid proteins in LSIL and HSIL as compared with detection of HPV L1 DNA. Acta Cytol. 2003;47:124-8.

35. Balan R, Simion N, Giusca SE, et al. Immunohistochemical assessment of p16, COX-2 and EGFR in HPV-positive cervical squamous intraepithelial lesions. Rom J Morphol Embryol. 2011;52:1187-94. 